\title{
Narratio et discours rapportés dans L'Heptaméron
}

\section{Véronique Montagne}

\section{(2) OpenEdition}

Journals

Édition électronique

URL : https://journals.openedition.org/rhetorique/1273

DOI : $10.4000 /$ rhetorique. 1273

ISSN : 2270-6909

\section{Éditeur}

UGA Éditions/Université Grenoble Alpes

\section{Édition imprimée}

ISBN : 978-2-37747-345-8

\section{Référence électronique}

Véronique Montagne, «Narratio et discours rapportés dans L'Heptaméron », Exercices de rhétorique [En ligne], 18| 2022, mis en ligne le 22 février 2022, consulté le 26 février 2022. URL : http:// journals.openedition.org/rhetorique/1273; DOI : https://doi.org/10.4000/rhetorique.1273

Ce document a été généré automatiquement le 26 février 2022.

\section{(c) (i) (2)}

Les contenus de la revue Exercices de rhétorique sont mis à disposition selon les termes de la Licence Creative Commons Attribution - Pas d'Utilisation Commerciale - Partage dans les Mêmes Conditions 4.0 International. 


\title{
Narratio et discours rapportés dans L'Heptaméron
}

\author{
Véronique Montagne
}

Dans un article intitulé "La lectio divina dans L'Heptaméron ", Christine MartineauGénieys proposait de considérer le recueil de Marguerite de Navarre comme un "roman dont les personnages se racontent des nouvelles", un "roman d'aventure spirituel, mis en route par la volonté même de Dieu ${ }^{1}$ ». Nous souscrivons entièrement à ce « renversement copernicien » d'un point de vue générique, tout en en précisant les enjeux pragmatiques : plus que de «nouvelles » à proprement parler, nous choisirons de parler ici de narrationes, au sens rhétorique du terme, c'est-à-dire de parties de la dispositio qui consistent à exposer « le déroulement des faits tels qu'ils se sont produits ou tels qu'ils peuvent s'être produits ${ }^{2}$ » dans le but d'exercer une certaine influence sur les auditeurs (devisants et lecteurs, en l'occurrence). La conversation conteuse de L'Heptaméron est effectivement souvent le lieu de l'expression d'opinions, par les devisants, lesquels s'opposent, se soutiennent, dans un débat d'idées régulièrement aporétique.

Chacune de ces narrationes se conforme globalement aux règles de l'exercice, à savoir la clarté, la brièveté et la vraisemblance, trois principes entérinés par la tradition rhétorique. Cette conformité souffre toutefois quelques exceptions, qui concernent les discours rapportés émaillant les récits. D'une longueur parfois remarquable ou tenus par des locuteurs inappropriés, ces discours rapportés relèvent à plusieurs reprises de ce que Raymond Lebègue a naguère qualifié d'" empire de la contio ", en constatant que « les propos que tiennent les personnages et par lesquels s'expriment leurs sentiments, sont trop composés et concertés ${ }^{3} »$. Dans un article sur la nouvelle 5, Nicole Cazauran a par ailleurs signalé que les villageois, sans doute aussi instruits que l'héroïne du conte, et qui, comme elle, "n'oyent quasi en l'an deux bons sermons ${ }^{4}$ ", sont toutefois capables de citer fidèlement saint Matthieu ou saint Luc $^{5}$. L'analyse proposée ici consistera à mettre en regard ces parties récurrentes de discours rapportés - dont la description énonciative, stylistique a été proposée ailleurs ${ }^{6}$ - avec les règles de la 
narratio, en nous attardant notamment sur le sens que l'on peut donner aux infractions régulières aux règles de celle-ci.

\section{Discours rapportés et règles de la narratio}

3 Avant d'entrer dans le détail des exceptions et du sens à donner à ces dernières, signalons que les discours rapportés respectent globalement les règles de la narratio notamment telles qu'elles sont rappelées par Pierre Fabri dans son Grant et vray art de pleine rethorique, la première rhétorique de langue française parue en 1534. La narratio y est définie comme la « seconde partie » d'un discours rhétorique,

qui est de la propre chose ou matiere subjecte ample \& longue exposition du cas que l'on veult soustenir ou deffendre en allegant hystoires auctoritez faict jugé ou choses semblables advenues ou fainctes estre advenues ou fables faictes à propos de la chose que l'on veult suader ou dissuader ${ }^{7}$.

Conformément à la tradition rhétorique, les règles de la narratio énumérées par Pierre Fabri sont la clarté ("premierement [que la narration] soit clerement et intelligiblement proferée »), la brièveté («secondement qu'elle soit brefve ») et la vraisemblance («tiercement, qu'elle soit vraye ou vray semblable, affin que les auditeurs la puissent croire $\left.^{8} »\right)$.

\section{a. Brièveté}

$4 \quad$ S'agissant des discours rapportés dans les nouvelles, le respect de la règle de la brièveté se lit dans le choix répété de la forme narrativisée dans le cas où il s'agit de propos qui ne méritent manifestement pas un développement conséquent ${ }^{9}$. Ainsi, dans la nouvelle 1 , les propos médisants du personnage féminin sont-ils rapidement résumés à l'aide du substantif «plainte» (« elle... en feit la plaincte à son evesque », p. 70). Le contenu des propos est parfois résumé dans un substantif locutoire comme "remonstrances" (« [elle] saillit dehors du lict en luy faisant toutes les remonstrances qu'il fut possible à femme de bien de luy faire », nouvelle 2, p. 78), « chanson » (" [elle] dist une chanson avec son mary », nouvelle 3, p. 82), «l'entreprinse » (« il declara à son amy l'entreprise qu'il avoit sur sa chambriere ", nouvelle 8, p. 108).

5 Par ailleurs, les propos qui se répètent sont rapportés au style indirect, à l'imparfait itératif et assortis des adverbes « souvent » ou « toujours » :

[Le] serviteur qu'elle avoit retenu [...] luy demandoit souvent, quand il seroit temps

d'aller querir son maistre. Elle luy respondit tousjours, qu'il viendroit assez tost.

(Nouvelle 1, p. 71)

Le discours direct constitue donc une forme d'amplificatio, une scène par rapport à la narration succincte des événements, ce qui signifie qu'il importe d'en justifier l'éventuelle longueur. La prolixité ne saurait aller de soi, ce qui explique que les personnages s'en étonnent ou que les devisants s'en dispensent. Ainsi, dans la longue nouvelle 10, Florinde souligne-t-elle qu'Amadour s'est longuement exprimé et, ce faisant, que cette prolixité fait probablement sens :

[...] pourquoy est-ce que vous me faictes une si grande et longue harangue?

(Nouvelle 10, p. 133)

Ainsi Parlamente signale-t-elle aussi qu'elle renonce à certains autres discours, qui sont manifestement de moindre importance dans la compréhension des enjeux du récit :

Je ne sçaurois entreprendre de vous peindre par le menu les propos qu'ilz eurent

pour rompre ceste jalousie [...]. (Nouvelle 10, p. 136) 
Je vous laisseray les propos que Florinde et Amadour eurent ensemble [...].

(Nouvelle 10, p. 142)

Cette pondération entre prolixité verbeuse et laconisme énigmatique correspond du reste à la brièveté telle qu'elle est définie dans un cadre rhétorique, où il est recommandé de n'en dire ni trop, ni trop peu. «La narration sera briefve se nous commençons à narrer là où il appartient sans rien dire de superfluité ou extravagant ne riens moins qu'il est besoing " précise ainsi Pierre Fabri ${ }^{10}$.

\section{b. Clarté}

7 Le respect du principe de clarté se lit dans le décryptage systématique de la véracité ou de la fausseté supposée des propos rapportés. L'auditeur / le lecteur est ainsi informé de la foi qu'il doit accorder à tel ou tel autre discours et le propos est rapporté sans qu'il ne puisse être abusé par un éventuel mensonge. C'est le rôle des indications de ton dans la nouvelle 1 :

Elle voyant que beaucoup de peuple estoit en l'eglise, et qu'il estoit accompaigné de deux bons serviteurs, se contraignit de parler le plus gracieusement qu'il luy fut possible [...]. (p. 71)

C'est le sens de la périphrase "donner à entendre " pour introduire un propos alors donné pour faux :

Et envoya à la court en diligence demander sa grace, donnant à entendre qu'il avoit plusieurs fois defendu sa maison à un personnage, dont il avoit suspicion qu'il pourchassoit le deshonneur de sa femme. (p. 73)

C'est encore le sens des éléments de caractérisation des différents locuteurs, qu'il s'agisse d'épithètes détachées («Elle, aussi sage et fine, qu'ils estoient fols et malicieux...», nouvelle 5, p.97) ou d'une relative périphrastique introduite par « comme » (« et comme celuy qui n'estoit despourveu d'invention, avec un visage pasle et transi, luy dist: "Helas! [....]" ", nouvelle 10, p. $153^{11}$ ) qui signalent pareillement, en l'occurrence, que les discours des personnages mis en scène contiennent des contrevérités.

\section{c. Vraisemblance}

9 Enfin, la narratio, discours rapportés inclus en l'occurrence, se doit d'être vraisemblable, « c'est-à-dire que le facteur doit dire parolles et choses que les auditeurs puissent croyre que il dye verité1 ${ }^{12} »$. Pierre Fabri précise que cela est possible lorsque le locuteur garde la «dignité de la personne ", ce qui correspond à la notion bien connue de decorum telle qu'elle est exposée dans l'Art poétique d'Horace, puis commentée par Jacques Peletier du Mans, Thomas Sébillet ou Antoine Fouquelin, entre autres auteurs alors intéressés par cette notion.

Mettre en scène des personnages vraisemblables, représentés selon les règles du decorum, cela signifie être attentif à la notion de caractère. À la dame d'honneur de la nouvelle 4 , sage et raisonnable, convient un discours où elle donne son avis de manière circonstanciée, en donnant, chemin faisant, une leçon de morale à sa jeune maîtresse ${ }^{13}$. À Florinde, jeune fille au début de la nouvelle 10, convient une réplique hésitante où elle témoigne de l'« ignorance joincte à [sa] jeunesse » (p. 133). À cette même Florinde, devenue femme, convient un discours plus assuré, dans lequel elle est capable de 
réutiliser les arguments auparavant employés par Amadour et de souligner les paradoxes du jeune homme ${ }^{14}$.

11 Cette convenance dicte le registre de langage : dans la nouvelle 8, Longarine place dans la bouche d'une «honneste femme de bien » des paroles emportées à l'égard de son mari adultère, propos dont la vivacité se traduit, entre autres, par le recours à une comparaison animalière polémique («vous eussiez prins une chevre coiffée pour une belle fille», p.111). Son époux, de condition semblable, utilise une exclamation familière, le « Ha vertu Dieu !» (p. 110) qui est censé convenir à son état.

\section{Discours rapportés et art oratoire}

12 Il arrive toutefois régulièrement que les discours rapportés soient composés de manière quelque peu artificielle, ou en tout cas qu'ils respectent avec brio les principes des discours oratoires en étant pourtant présentés comme des interventions spontanées. On en prendra ici pour preuve l'intervention de la dame d'honneur dans la nouvelle 4 , qui relève du genre délibératif, en renvoyant le lecteur à une étude publiée en $2006^{15}$.

13 On s'intéressera aussi au discours de Rolandine dans la nouvelle 21, lequel témoigne d'un art consommé du genre épidictique. La jeune femme s'y défend en insistant sur la faute de sa parente, la Reine, et la liste des torts qu'elle lui impute est considérable: une sévérité excessive ( vous, ma dame (qui jamais ne me pardonnastes une seule petite faulte, ne me loüastes de nul bon œuvre)... », p. 264), un pouvoir de nuisance qui s'étend à plusieurs personnes ( «la mauvaise volonté que de long temps avez portée à monsieur mon pere et à moy [...]», p. 263) et qui s'étend d'un point de vue temporel (" que de long temps avez portée... », p. 263 ; « vous, ma dame, avez grande occasion de pleurer tant pour le grand tort qu'en toute ma jeunesse m'avez tenu, que pour celuy que maintenant vous me faictes [...] », p. 266). Rolandine y manifeste aussi une maitrise du genre judiciaire en opérant un habile transfert des responsabilités (« une faulte, qui doit estre imputée plus à vous qu'à moy [...]», p. 266) et en accusant son interlocutrice du vice d'injustice ("vous m'avez laissée comme une personne oubliée du tout en vostre bonne grace [...]», p. 263 ; «le peu d'estime qu'avez faict de moy [...]», p. 263; « vous me tenez et faictes grand tort [...]», p. 264).

On ajoutera enfin que la jeune femme de la nouvelle 15 prononce un discours révélant une grande maîtrise du genre judiciaire, lequel « comprend l'accusation et la défense ", et porte sur «le passé, puisque c'est toujours sur des faits accomplis que porte l'accusation ou la défense ${ }^{16}$ ». La jeune femme s'y oppose aux accusations de son mari, en un long discours au terme duquel elle lui demande de "juger sans faveur » de la gravité de leurs torts respectifs. Il lui est impossible de nier les faits accomplis, car ils sont connus de son interlocuteur:

Je ne veulx poinct justifier, monsieur, ne faire moindre envers vous l'amour que j'ay portée au gentilhomme, dont vous avez soupçon : car vous ne le pouvez ny ne devez croire, veu l'experience qu'aujourd'huy en avez euë [...]. (p. 206)

Elle choisit donc de se défendre en transférant la responsabilité de son acte sur son accusateur, procédé également utilisé par Rolandine ${ }^{17}$. C'est un procédé du discours judiciaire bien décrit par l'auteur de Rhétorique à Herennius ${ }^{18}$ ou par Quintilien, qui précise qu'on peut attaquer directement la victime elle-même, qui est ici le mari qui se plaint du comportement de sa femme ${ }^{19}$. La jeune femme compare ainsi, point par point, 
sa propre situation et celle de son époux. Comme la Rhétorique à Herennius le recommande à tout accusé, elle insiste sur le fait qu'elle « a failli par ignorance ${ }^{20}$ »:

\begin{tabular}{|c|c|}
\hline Victime & Accusée \\
\hline $\begin{array}{l}\text { "vous estes homme sage, et experimenté, et d'aage, pour } \\
\text { cognoistre et sçavoir eviter le mal » }\end{array}$ & $\begin{array}{l}\text { "moy jeune et sans experience } \\
\text { nulle, de la force et puissance } \\
\text { d'amour» (p. 208) }\end{array}$ \\
\hline $\begin{array}{l}\text { "Vous avez une femme qui vous cherche, estime, et aime } \\
\text { plus que sa vie propre" }\end{array}$ & $\begin{array}{l}\text { "j'ay un mary qui me fuit, qui me } \\
\text { hait, et me despite plus qu'une } \\
\text { chambriere» (p. 208) }\end{array}$ \\
\hline $\begin{array}{l}\text { "Vous aimez une femme desja d'aage, et en mauvais point, } \\
\text { et moins belle que moy" }\end{array}$ & $\begin{array}{l}\text { «j'aime un gentil-homme plus jeune } \\
\text { que vous, plus beau et plus } \\
\text { amiable» (p. 208) }\end{array}$ \\
\hline $\begin{array}{l}\text { "Vous aimez la femme d'un des plus grands amis que vous } \\
\text { ayez en ce monde, et l'amye de vostre maistre, offençant } \\
\text { d'un costé l'amitié et de l'autre la reverence que vous portez } \\
\text { à tous deux" }\end{array}$ & $\begin{array}{l}\text { "j'aime un gentilhomme qui n'est à } \\
\text { rien lié, sinon à l'amour qu'il me } \\
\text { porte» (p. 208) }\end{array}$ \\
\hline
\end{tabular}

Indépendamment de ces quelques cas de longs discours manifestant une maîtrise de l'art oratoire, les personnages des récits recourent à tous les types de raisonnement, qu'il soit déductif :

Je ne puis pas (dict elle) avoir l'honneur et le plaisir ensemble : Je sçay bien que j'ay

l'honneur dont une reçoit le plaisir : aussi celle qui a le plaisir, n'a pas l'honneur que j'ay. (p. 83)

Ou inductif :

Ma dame, j'ay à vous faire un beau compte [...]. (p. 531)

Ils utilisent tous les lieux de l'art oratoire : l'argument d'autorité («les poëtes disent que les dieux se rient des juremens [...]», p. 217); l'argument pragmatique ("Je conseilleray tousjours à mes amis de parler et non de mourir, car il y a peu de parolles qui ne se puissent amender, mais la vie perduë ne se peut recouvrer.», p.131); l'argument de direction (« [Ils] dirent qu'ils ne cherchoient que sa bonne grace en leurs enchantements [...] », p. 76); l'argument a fortiori (« si moy qui suis vostre maistre vous porte telle affection, que pour le moins ne me la sçauriez porter moindre », p. 168) ou encore la règle de justice (« Si vous aimez ma vie, aussy feray-je la vostre. », p. 169).

17 Ils utilisent tous les ressorts de la réfutation, réfutation des propos de Florinde par Amadour dans la nouvelle 10 :

Donc, dist Amadour, puisque ne me voulez faire vivre, pourquoy me voulez-vous garder de morir? (p. 156)

Réfutation du mari de la nouvelle 35 par sa femme qui recourt à l'argumentation par la dissimilitude ${ }^{21}$ :

Ils sont bons en l'eglise et aux predications, mais aux maisons sont Antechrists.

(p. 373)

18 Comment croire, alors, à la spontanéité de discours et de raisonnements aussi travaillés et comment, par conséquent, considérer que les discours de ces personnages 
sont crédibles? Comment donc penser ces manquements réguliers à la règle de vraisemblance qui est celle de la narratio?

\section{Fonctions des discours rapportés}

19 Comme le fait observer Gillian Lane-Mercier au sujet de cas similaires de discours rapportés, si les quelques exemples pointés ici sont de ceux qui « miment mal la réalité, en revanche ils empruntent de façon générale une direction parallèle à l'orientation diégétique, et remplissent une pluralité de fonctions qui complètent le travail narratif comme tel ${ }^{22}$ ». C'est de cette « direction parallèle » dont il sera question dans les lignes qui suivent, où seront évoquées les notions d'orientation argumentative d'une part, de convenance oratoire d'autre part.

\section{a. Un outil argumentatif}

Dans La Parole pamphlétaire, Marc Angenot définit l'intéressante catégorie du «témoin fictif » en ces termes :

[...] l'argumentateur fait appel à un témoin imaginaire, censé objectif, et donc situé dans une autre époque ou une autre culture. Il s'agit d'une sorte d'argument d'autorité par fiction ${ }^{23}$.

21 Les personnages que les devisants font parler sont des exemples de ces témoins fictifs, parce qu'ils leur servent à dégager la morale du récit, et/ou parce qu'ils sont le porteparole des convictions du locuteur qui les anime.

Il arrive que la morale du conte soit ainsi proposée par un des personnages, la péroraison du locuteur n'en semblant ainsi que plus objective. Dans la nouvelle 2, Oisille délègue ainsi à l'un de ses personnages le rôle d'étayer le credo qu'elle soutient : "[elle] rendit graces à son Dieu, lequel elle nommoit sa force, sa vertu, sa patience et chasteté [...] » (p. 79). Dans la nouvelle 5, la condamnation des prêtres par les villageois aux propos étonnamment érudits, prépare celle que fait Guebron des deux prêtres, "deux si malicieux hommes» (p. 100). Dans la nouvelle 3, un éloquent gentilhomme persuade une reine des bienfaits de l'adultère comme le fait ensuite Saffredent en concluant son récit (p. 88). Dans la nouvelle 4, enfin, la plainte lyrique du gentilhomme («O beauté ! [...]», p.92) épouse le point de vue d'Emarsuitte, narratrice de cette nouvelle.

\section{b. Retour sur la notion de convenance}

23 Par voie de conséquence, si les paroles des personnages ne conviennent pas nécessairement à ces derniers, s'ils ne relèvent pas du respect du decorum évoqué plus haut ${ }^{24}$, ils correspondent en revanche au credo du narrateur qui les anime. Les exemples de Saffredent, Dagoucin, et Oisille illustrent cette délégation de conviction à des porte-parole.

24 Saffredent raconte la nouvelle 3, dans laquelle un gentilhomme essaie de séduire une reine. Il lui attribue un discours où l'on retrouve ses propres stratégies oratoires, qu'il déploie durant les différents débats avec les autres devisants : 


\begin{tabular}{|c|c|c|}
\hline $\begin{array}{l}\text { Type ou } \\
\text { contenu des } \\
\text { arguments }\end{array}$ & $\begin{array}{l}\text { le gentilhomme de la } \\
\text { nouvelle } 3(\text { p. 83-84) }\end{array}$ & Saffredent \\
\hline $\begin{array}{l}\text { Se servir du } \\
\text { pathos }\end{array}$ & $\begin{array}{l}\text { «[Il] print la hardiesse, un jour } \\
\text { en parlant à la Royne, de luy } \\
\text { dire, qu'il avoit grande pitié de } \\
\text { ce qu'elle n'estoit autrement } \\
\text { aymée du Roy son mary» }\end{array}$ & $\begin{array}{l}\text { «le diable, voyant que tous les tourmens } \\
\text { d'enfer ne me pouvoient faire pis que ceux } \\
\text { qu'elle me donnoit, ne me daigna jamais } \\
\text { prendre» (p. 304) }\end{array}$ \\
\hline $\begin{array}{l}\text { Commencer par } \\
\text { l'honneur }\end{array}$ & $\begin{array}{l}\text { «Ma dame, l'honneur est né avec } \\
\text { vous » }\end{array}$ & $\begin{array}{l}\text { «qui est celle qui fermera ses aureilles, quand } \\
\text { nous commencerons à l'honneur et à la } \\
\text { vertu?»(p. 175) }\end{array}$ \\
\hline $\begin{array}{l}\text { Évoquer } \\
\text { «loi } \\
\text { nature » }\end{array}$ & $\begin{array}{l}\text { «si le Roy avoit mis sa couronne } \\
\text { hors de dessus sa teste, je pense } \\
\text { qu'il n'auroit nul advantage sur } \\
\text { moy de contenter une dame. } \\
\text { Estant seur que pour satisfaire à } \\
\text { une si honneste personne que } \\
\text { vous, il devoit vouloir avoir } \\
\text { changé sa complexion à la } \\
\text { mienne» }\end{array}$ & $\begin{array}{l}\text { "Pensez [...] que voilà une bonne et sage } \\
\text { femme, qui, pour se monstrer plus vertueuse } \\
\text { par dehors, qu'elle n'estoit au cueur, et pour } \\
\text { dissimuler une amour, que la raison de nature } \\
\text { vouloit qu'elle portast à un si honneste } \\
\text { seigneur, se laissa mourir, par faulte de se } \\
\text { donner le plaisir qu'elle desiroit } \\
\text { couvertement» (p.327) }\end{array}$ \\
\hline $\begin{array}{l}\text { Se servir de la } \\
\text { religion dans } \\
\text { cette } \\
\text { casuistique } \\
\text { amoureuse }\end{array}$ & $\begin{array}{l}\text { «je sçay bien que l'honneste } \\
\text { amour de vostre cueur vous } \\
\text { rendroit tel contentement s'il } \\
\text { trouvoit en celuy du Roy pareil } \\
\text { amour: mais Dieu vous en a bien } \\
\text { gardée, à fin que ne trouvant en } \\
\text { luy ce que vous demandez, vous } \\
\text { n'en feissiez vostre Dieu en } \\
\text { terre» }\end{array}$ & $\begin{array}{l}\text { "je croy que Dieu ne se courrouce poinct de } \\
\text { tel peché, veu que c'est un degré pour monter } \\
\text { à l'amour parfaicte de Dieu, où jamais nul ne } \\
\text { peut monter facilement, qui n'ait passé par } \\
\text { l'eschelle de l'amour de ce monde" } \\
\text { (p. 381-382) }\end{array}$ \\
\hline
\end{tabular}

Il en va de même chez Dagoucin, qui fait dire aux gentilshommes des nouvelles 9 et 24 des choses qu'il ne désavouerait pas :

\begin{tabular}{|l|l|l|l|}
\hline $\begin{array}{l}\text { Type ou } \\
\text { contenu des } \\
\text { arguments }\end{array}$ & $\begin{array}{l}\text { Gentilshommes (nouvelle 9, } \\
\text { p. 117-118 et nouvelle 24,p. 197) }\end{array}$ & Dagoucin \\
\hline & $\begin{array}{l}\text { "tant qu'il m'a esté possible, j'ay } \\
\text { dissimulé l'amour que je porte à ma } \\
\text { damoiselle vostre fille... (cause } \\
\text { La nécessité de } \\
\text { cacher } \\
\text { amour }\end{array}$ & $\begin{array}{l}\text { "j'ay si grand peur que la demonstrance } \\
\text { face tort à la perfection de mon amour, } \\
\text { aimé mourir que desirer une seule } \\
\text { chose qui eust esté à son } \\
\text { deshonneur ») }\end{array}$ & $\begin{array}{l}\text { que je crains que celle de qui je devrois } \\
\text { desirer amitié semblable, l'entende » } \\
\text { (p. 114) }\end{array}$ \\
\hline
\end{tabular}




\begin{tabular}{|l|l|l|}
\hline & «Le temps m'a faict veoir sur quel & «si nostre amour est fondée sur la \\
Leaulté, bonne grace, amour et faveur \\
fondements & fondement / Mon cueur vouloit aymer & si fermement: / Ce fondement estoit \\
de l'amour & vostre beauté / [...] Le temps m'a faict & longuement durer. Car si la chose surquoy \\
& veoir beauté estre rien » & $\begin{array}{l}\text { nous la fondons deffault, nostre amour } \\
\text { s'en volle hors de nous » (p. 114) }\end{array}$ \\
\hline
\end{tabular}

Au début de la sixième journée, l'ainée du groupe affirme qu'elle racontera la nouvelle 51 avec une intention comparable à celle qui l'anime lors de la lectio divina (p. 138). Elle n'a pas attendu ce jour pour glisser dans ses nouvelles, et plus spécifiquement dans les propos de ses personnages, des remarques qui vont dans le sens de la morale qu'elle ne cesse de prêcher à ses compagnons. Les nouvelles 2 et 70 en témoignent, selon le même principe que chez Saffredent ou Dagoucin :

\begin{tabular}{|c|c|c|}
\hline $\begin{array}{l}\text { Type et } \\
\text { contenu des } \\
\text { arguments }\end{array}$ & $\begin{array}{l}\text { Les femmes des nouvelles } 2 \\
\text { (p. 78-80) et } 70 \text { (p. 577) }\end{array}$ & Oisille \\
\hline $\begin{array}{l}\text { Le Christ } \\
\text { rédempteur }\end{array}$ & $\begin{array}{l}\text { «son filz, auquel elle croyoit } \\
\text { fermement tous ses pechez } \\
\text { estre lavez, et effacez de la } \\
\text { memoire de son ire» }\end{array}$ & $\begin{array}{l}\text { «la volonté de Dieu, qui pour nous a envoyé son } \\
\text { fils en terre annoncer ceste saincte parolle et } \\
\text { bonne nouvelle, par laquelle il promet remission } \\
\text { des pechez » (p. 63) }\end{array}$ \\
\hline La grâce & $\begin{array}{l}\text { "Seigneur, recevez l'ame qui } \\
\text { par vostre bonté a esté } \\
\text { racheptée!» }\end{array}$ & $\begin{array}{l}\text { «les graces de Dieu ne se donnent point aux } \\
\text { hommes, pour leur noblesse et richesses, mais } \\
\text { selon qu'il plaist à sa bonté... » (p. } 80)\end{array}$ \\
\hline $\begin{array}{l}\text { Dieu, source } \\
\text { fiable du « vrai » }\end{array}$ & $\begin{array}{l}\text { «O mon Dieu mon Createur! } \\
\text { qui estes le vray et parfaict } \\
\text { amy ; « en vous seul j'ay ma } \\
\text { parfaicte confiance» }\end{array}$ & $\begin{array}{l}\text { « il n'y a nulle, qui ne sçache bien ce qu'elle doit } \\
\text { croire, c'est de jamais ne mettre en doute la } \\
\text { parole de Dieu, et moins adjouster foy à celle des } \\
\text { hommes. » (p. 504) }\end{array}$ \\
\hline
\end{tabular}

Les narrationes faites par les devisants de L'Heptaméron ont pour but de soutenir une idée, un positionnement, un éclairage subjectif sur les relations entre les hommes et les femmes ou sur le monde comme il va. L'efficacité prévaut et si infractions il semble y avoir aux règles de la narratio, celles-ci s'expliquent par l'orientation argumentative de chacun des récits. Pour faire adhérer à une opinion, on ne saurait être verbeux, imprécis, invraisemblable dans les exemples (au sens rhétorique du terme) qui sont rapportés. Dans l'entreprise polyphonique qui est celle de L'Heptaméron, où des personnages-devisants parlent à d'autres personnes qui sont elles-mêmes tour à tour devisants ou auditeurs, pendant que des moines, tapis, écoutent aussi quelquefois les récits, les personnes qui monopolisent le précieux espace discursif ne sauraient parler en vain : ils sont porteurs d'enseignements divers, approuvés ou réprouvés par le reste de la communauté, dans un franc parler qui les rend crédibles et vivants. 


\section{NOTES}

1. C. Martineau-Génieys, "La lectio divina dans l'Heptaméron", dans Études sur l'Heptaméron de Marguerite de Navarre, Colloque de Nice, février 1992, Université de Nice-Sophia Antipolis, 1996, p. 42.

2. Rhétorique à Herennius, Paris, Les Belles Lettres, 2003, I, 3, 4, p. 4.

3. R. Lebègue, « Réalisme et apprêt dans la langue des personnages de l'Heptaméron », La littérature narrative d'imagination, Colloque de Strasbourg, 23-25 avril 1959, Paris, PUF, 1961, p. 80.

4. Marguerite de Navarre, L'Heptaméron, éd. N. Cazauran et S. Lefèvre, Paris, Gallimard (Folio classique), 2000, p. 100. Les citations suivantes sont extraites de cette édition.

5. Voir N. Cazauran, «À propos de l'Heptaméron. Lecture de la cinquième nouvelle ", Littératures, 1991, n² 25, p. 17-28 et «Les citations bibliques dans l'Heptaméron », dans Variétés pour Marguerite de Navarre 1978-2004, Paris, Honoré Champion, 2005, p. 379-391.

6. M.-C. Thomine et V. Montagne, Marguerite de Navarre, L'Heptaméron, Neuilly, Atlande, 2020, p. 303-328.

7. Pierre Fabri, Le Grant et vray art de pleine rethorique, Paris, Denis Janot, $1534, \mathrm{f}^{\circ} \mathrm{xxxv} \mathrm{v}^{\circ}$ et $\mathrm{xxxvi}$ $\mathrm{r}^{\circ}$.

8. Ibid., $\mathrm{f}^{\circ} \mathrm{xxxvi} \mathrm{v}^{\circ}$.

9. Dans La Représentation du discours autre: principes pour une description, Berlin/ Boston, De Gruyter, 2020, p. 243, Jacqueline Authier-Revuz précise ainsi que « le recours à la spécification du thème du dire (de préférence à une reformulation de son contenu) est [...] la forme privilégiée des représentations ironiques, polémiques de propos qui - stéréotypés, répétitifs, logorrhéiques ne "méritent pas" qu'on aille au-delà de leur thème ".

10. Pierre Fabri, op. cit., fo xxxvii $\mathrm{r}^{\circ}$.

11. Sur ces relatives, voir notre article «De l'usage des relatives périphrastiques dans L'Heptaméron de Marguerite de Navarre", Le Verger, Revue du site électronique Cornucopia, Bouquet XX, dirigée par G. Amiel, A. Boutet, A.-G. Leterrier-Gagliano et A. Lionetto, 2021 (http:// cornucopia16.com/blog/2021/01/26/bouquet-xx-lheptameron-de-marguerite-de-navarre/).

12. Pierre Fabri, op. cit., f $\mathrm{f}^{\circ} \mathrm{xxvii} \mathrm{v}^{\circ}$.

13. Voir notre article «Le discours délibératif et épidictique d'une dame d'honneur. Étude stylistique de la Nouvelle 4 ", dans Lire L'Heptaméron de Marguerite de Navarre, ClermontFerrand, PU Blaise Pascal, collection CERHAC, 2006, p. 133 sq.

14. Dans la nouvelle 10, Amadour explique à Floride quelles ont été ses motivations en recourant à une comparaison architecturale, tirée de l'évangile selon Matthieu $(7,26-27)$ : « celuy qui veult bastir un edifice perpetuel, doit regarder un seur et ferme fondement : parquoy moy qui desire perpetuellement demeurer en vostre service, je regarde, non seulement les moyens de me tenir près de vous, mais d'empescher que l'on ne puisse congnoistre la grande affection que je vous porte» (p.134). Devenue adulte, la jeune femme reprend ce thème sous forme métaphorique, afin d'en montrer les limites à Amadour : «j'estois venuë icy, deliberée de prendre un tresseur fondement, mais Amadour, en un moment m'avez monstré, qu'en lieu d'une pierre nette et pure, le fondement de cest edifice est assis sur un sablon leger et mouvant, ou sur la fange molle et infame » (p. 146-147).

15. Voir la note 12.

16. Aristote, Rhétorique, I, III, 3, Paris, Le Livre de poche, 1991, p. 93-94.

17. Voir supra.

18. Rhétorique à Herennius, II, 22, op. cit., p. 53 : «Il y a transfert de responsabilité quand l'accusé impute la raison de son acte à une faute commise par autrui ». 
19. Quintilien, Institution oratoire, VII, 4, 7-8, Paris, Panckoucke, 1831, p. 343 : «[Un] genre de défense se prend, non dans le fait, s'il n'est pas justifiable par lui-même, mais dans les circonstances extérieures [...]. En ce genre, ce qu'il y a de plus efficace, c'est de justifier un crime par les motifs qu'on a eu de le commettre [...] et c'est là proprement une récrimination, [...] puisqu'on ne défend qu'en accusant sa victime ».

20. Rhétorique à Herennius, II, 23, op. cit., p. 55.

21. Cicéron, De l'invention, Paris, Les Belles Lettres, 1994, I, XLIV, 82, p. 121-122: «Lorsque l'adversaire introduira un élément de comparaison, [...] il conviendra dans la réfutation de dire que la chose comparée n'est pas semblable à l'élément de comparaison. ».

22. G. Lane-Mercier, La Parole romanesque, Paris, Klincksieck, 1989, p. 36.

23. M. Angenot, La Parole pamphlétaire, Paris, Payot, 1982, p. 204.

24. Voir supra.

\section{AUTEUR}

\section{VÉRONIQUE MONTAGNE}

Université Côte d'Azur, Laboratoire CNRS BCL UMR 7320 